\title{
Integrative radiogenomics for virtual biopsy and treatment monitoring in ovarian cancer
}

Paula Martin-Gonzalez ${ }^{1,2}$, Mireia Crispin-Ortuzar ${ }^{1,2}$, Leonardo Rundo 2,3, Maria Delgado-Ortet ${ }^{2,3}$, Marika Reinius ${ }^{1,2}$, Lucian Beer ${ }^{2,3,4}$, Ramona Woitek ${ }^{2,3,4}$, Stephan Ursprung ${ }^{2,3}$, Helen Addley ${ }^{1,3,5}$, James D. Brenton 1,2, Florian Markowetz ${ }^{1,2}$ and Evis Sala $2,3^{*}$ (D)

\begin{abstract}
Background: Ovarian cancer survival rates have not changed in the last 20 years. The majority of cases are Highgrade serous ovarian carcinomas (HGSOCs), which are typically diagnosed at an advanced stage with multiple metastatic lesions. Taking biopsies of all sites of disease is infeasible, which challenges the implementation of stratification tools based on molecular profiling.

Main body: In this review, we describe how these challenges might be overcome by integrating quantitative features extracted from medical imaging with the analysis of paired genomic profiles, a combined approach called radiogenomics, to generate virtual biopsies. Radiomic studies have been used to model different imaging phenotypes, and some radiomic signatures have been associated with paired molecular profiles to monitor spatiotemporal changes in the heterogeneity of tumours. We describe different strategies to integrate radiogenomic information in a global and local manner, the latter by targeted sampling of tumour habitats, defined as regions with distinct radiomic phenotypes.

Conclusion: Linking radiomics and biological correlates in a targeted manner could potentially improve the clinical management of ovarian cancer. Radiogenomic signatures could be used to monitor tumours during the course of therapy, offering additional information for clinical decision making. In summary, radiogenomics may pave the way to virtual biopsies and treatment monitoring tools for integrative tumour analysis.
\end{abstract}

Keywords: Radiomics, Radiogenomics, Ovarian cancer, Tumour habitats, Virtual biopsies

\footnotetext{
* Correspondence: es220@medschl.cam.ac.uk

${ }^{2}$ Cancer Research UK Cambridge Centre, University of Cambridge, Cambridge CB2 ORE, UK

${ }^{3}$ Department of Radiology, University of Cambridge, Cambridge CB2 0QQ, UK Full list of author information is available at the end of the article
}

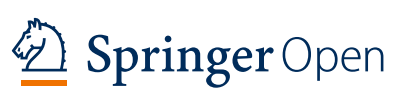

(c) The Author(s). 2020 Open Access This article is licensed under a Creative Commons Attribution 4.0 International License which permits use, sharing, adaptation, distribution and reproduction in any medium or format, as long as you give appropriate credit to the original author(s) and the source, provide a link to the Creative Commons licence, and indicate if changes were made. The images or other third party material in this article are included in the article's Creative Commons licence, unless indicated otherwise in a credit line to the material. If material is not included in the article's Creative Commons licence and your intended use is not permitted by statutory regulation or exceeds the permitted use, you will need to obtain permission directly from the copyright holder. To view a copy of this licence, visit http://creativecommons.org/licenses/by/4.0/. 


\section{Key points}

- Radiogenomics is the integration of radiomics and genomics and holds the potential to improve treatment response and outcome prediction in ovarian cancer.

- Creating virtual biopsies to overcome the limitations of invasive biopsies may be feasible due to recent technological advances that allow integration of imaging and molecular data.

- Radiogenomics studies that ensure reproducible, tissue-matched radiomic signatures in large prospective cohorts are needed for the clinical implementation to become a reality.

\section{Background}

Ovarian cancer is the second most prevalent of gynaecological cancers [1]. While survival rates for most tumours have improved over the last decades, the 5-year survival rate of ovarian cancer has not changed since 1980 [2]. The majority of the ovarian tumours are highgrade serous ovarian carcinomas (HGSOCs) [2, 3] which are characterised by a high degree of heterogeneity that manifests on multiple levels. Biologically, HGSOC is a solid tumour driven by recurrent point mutations in TP53 and BRCA $1 / 2$ and by DNA copy number alterations [4-6]. Copy number changes arising from a variety of mutational processes, such as insertion, deletion or duplication of chromosomal fragments, are thought to allow for growing tissues to adapt to environmental selection forces [7]. Additionally, tumour microenvironment (TME) heterogeneityi.e., the varying proportions of tumour, stroma and immune cells, as well as ascites components, such as cytokines and growth factors-is known to be a hallmark of HGSOC. The interplay of these factors is yet to be understood [8].

Clinically, HGSOC is frequently diagnosed as an advanced multi-site disease with lesions in the pelvis and peritoneal cavity [9]. The standard of care treatment is either primary debulking surgery followed by adjuvant chemotherapy, or neoadjuvant chemotherapy (NACT) followed by delayed primary surgery. HGSOC is particularly sensitive to platinum-based NACT due to defects in homologous recombination $[10,11]$. However, treatment resistance develops in up to $90 \%$ of patients over time and is the main cause of death in HGSOC $[12,13]$. The main hypothesis for the resistance mechanism is the presence of tumour cell clones with different genomic profiles that evolve to acquire treatment resistance [14-16]. Genomic changes are also linked to the heterogeneity of the TME, which may impose different selective pressures on clonal populations [17-20].
In order to improve the management of patients with HGSOC, new tools are required to enable earlier detection of resistance to chemotherapy and evidence-based therapeutic stratification. The main challenge for the implementation of such tools resides in accurately characterising the multi-scale complexity of the disease, across tumour regions and metastatic sites. So far, the main approach to evaluate intra- and inter-lesion heterogeneity has been multiple sampling. However, this approach is invasive, expensive and impractical and may still provide inadequate coverage of the disease depending on sample location [21].

Tools based on standard of care or advanced radiological imaging, both at diagnosis and throughout the course of therapy, may capture the spatiotemporal tumour complexity in a comprehensive and non-invasive manner [22]. This information can be useful to predict the probability of certain molecular profiles of interest, by obtaining virtual biopsies from radiological images, and allowing for real biopsies to be taken in a more informed way.

In the following sections, we first describe how radiomic analysis, the quantitative analysis of medical images, can be integrated with molecular information via radiogenomic studies. We then discuss the importance of tumour habitats, defined as regions with local distinct radiomic features, in this integrative process. We also outline technological advances to take habitat-specific samples to integrate molecular profiles and radiomics in a spatial manner. This may enable the creation of prediction maps for molecular profiles, so-called "virtual biopsies". An overview of this process is presented in Fig. 1.

\section{Main text \\ From radiomics to radiogenomics: comprehensive measures of tumour biology}

Medical imaging, primarily computed tomography (CT), is crucial for diagnosing HGSOC, evaluating its extent and assessing treatment response [23, 24]. Although current routine evaluation is mostly semantic and qualitative [25], it has become widely accepted that mining images in a quantitative manner may add useful information for clinical decision-making [26].

The analysis of quantitative features extracted from imaging data on a large scale is commonly referred to as radiomics [27-29]. Radiomic studies exploit the fact that medical imaging encodes information of the underlying tissues and aims to define quantitative imaging biomarkers related to clinical endpoints [30]. The typical radiomics workflow consists of five steps performed on images of different modalities, usually CT, positron emission tomography (PET) or magnetic resonance 


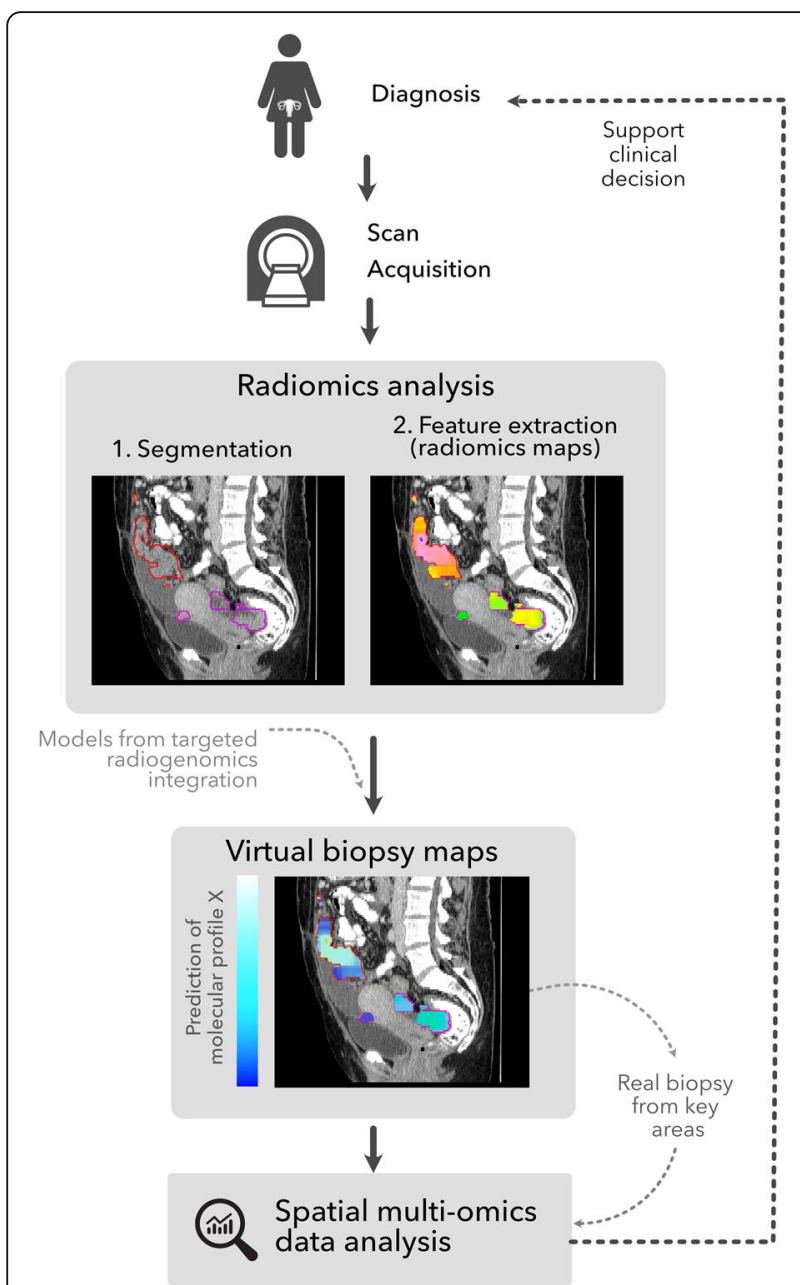

Fig. 1 Overview of the workflow leading to the creation of virtual biopsy maps that can be used, together with real time biopsies from key areas, to inform clinical decisions. These virtual biopsy maps offer the possibility to be calculated at different stages of the clinical process-e.g., between chemotherapy cycles - to study the spatiotemporal variation of the molecular profiles

imaging (MRI). The steps are (i) formulation of the clinical question and selecting the most appropriate type of imaging, (ii) tumour segmentation, (iii) image preprocessing, (iv) feature extraction from tumour regions (e.g., intensity, shape, volume and texture on original or filtered scans) and (v) predictive modelling of clinical endpoints through machine learning algorithms-including feature selection, model training and validation of the findings in internal or external datasets [31]. These steps and their limitations have been discussed in the existing literature [26, 28, 30-34]. More recently, radiomic analyses have also included deep learning methods, which have the advantage of being able to learn the most useful quantitative representations of the data by themselves, therefore bypassing the need for handcrafted features [35-37].

Radiomic studies have focused on understanding treatment response, recurrence and survival [38, 39]. Conceptually, the hypothesis underlying these analyses is that different imaging phenotypes capture the pathophysiology of the lesions [29]. The study of the association between radiomic and biological features, particularly genomics, is usually called imaging genomics or, more commonly, radiogenomics [40]. The field is still in its early stages, but initial results are promising.

Although limited radiogenomics literature is available in HGSOC, several studies showed a significant association of radiomics with relevant molecular profiles (Table 1). As discussed above, one of the most frequent mutations in HGSOC patients is in the BRCA1 or BRCA2 genes [49]. Patients with BRCA mutations tend to have better responses to chemotherapy due to the higher platinum sensitivity, and this improves their overall survival when compared to BRCA wild-type patients [50,51]. Using features from CT in a dataset with 108 HGSOC patients, a higher likelihood of having mutant BRCA was found to be related with nodular pelvic disease and the presence of disease in the gastrohepatic ligament, whilst infiltrative pelvic disease, mesenteric presence and supradiaphragmatic lymphadenopathy were related with decreased likelihood of BRCA mutation [42]. Additionally, the same study reported the higher likelihood of incomplete resection in BRCA wild-type HGSOC in cases with mesenteric involvement and lymphadenopathy in supradiaphragmatic, as well as shorter PFS in both mutant and wild-type BRCA patients with suprarenal para-aortic regions and pelvic disease presence in the lesser sac and left upper quadrant and the mesenteric involvement relationship [42].

Looking also at the genomic level, a high-resolution genome-wide study of copy number variations in 118 ovarian cancer patients revealed the association of 19q12 amplification, which contains the cyclin E1 gene (CCNE1), with failure of primary treatment and worse survival [52]. CCNE1 is known to play a role in different processes of tumour cells, such as G1 to $\mathrm{S}$ phase transition, replication of DNA, apoptosis and chromosomal instability [53]. Using CT-based radiomic analysis of 38 HGSOC patients, Vargas and coauthors [43] reported that high variation of texture features between lesions-described as high inter lesion heterogeneity-was associated with the amplification of $19 \mathrm{q} 12$.

Using transcriptomic information from The Cancer Genome Atlas (TCGA) [54], four subtypes of 
Table 1 Summary of radiogenomics papers focusing on High Grade Serous Ovarian Cancer (HGSOC). CLOVAR refers to the Classification of Ovarian Cancer proposed in [41]

\begin{tabular}{|c|c|c|c|c|c|c|}
\hline Method & Ref. & Patients & Imaging & $\begin{array}{l}\text { Level of } \\
\text { association }\end{array}$ & Biological correlate & Results \\
\hline \multirow[t]{6}{*}{$\begin{array}{l}\text { Regular } \\
\text { association }\end{array}$} & {$[42]$} & 108 & $C T$ & Genomics & BRCA mutation & $\begin{array}{l}\text { Nodular pelvic disease and the presence of pelvic disease in the } \\
\text { gastrohepatic ligament associated with high likelihood of BRCA. } \\
\text { Infiltrative pelvic disease, mesenteric presence and supradiaphragmatic } \\
\text { lymphadenopathy were related with less likelihood of BRCA mutation. }\end{array}$ \\
\hline & {$[43]$} & 38 & $\mathrm{CT}$ & Genomics & 19q12 amplification & $\begin{array}{l}\text { High inter lesion heterogeneity is associated with the amplification } \\
\text { of } 19 q 12 .\end{array}$ \\
\hline & {$[44]$} & 46 & $C T$ & Transcriptomics & $\begin{array}{l}\text { CLOVAR } \\
\text { classification }\end{array}$ & $\begin{array}{l}\text { Peritoneal disease and mesenteric infiltration related with the } \\
\text { mesenchymal subtype of the CLOVAR. }\end{array}$ \\
\hline & {$[45]$} & 92 & $\mathrm{CT}$ & Transcriptomics & $\begin{array}{l}\text { CLOVAR } \\
\text { classification }\end{array}$ & $\begin{array}{l}\text { Peritoneal involvement and presence of disease in the pelvis and ovary } \\
\text { related with mesenchymal subtype. }\end{array}$ \\
\hline & {$[46]$} & 20 & $\mathrm{CT}$ & Proteomics & Selected proteins & $\begin{array}{l}\text { Intra- and inter-site heterogeneity associated with selected proteins } \\
\text { involved in aminoacid metabolism. }\end{array}$ \\
\hline & {$[47]$} & 297 & $\mathrm{CT}$ & Multilevel & $\begin{array}{l}\text { DNA damage and } \\
\text { stromal phenotype }\end{array}$ & $\begin{array}{l}\text { CT-based radiomic signature with prognostic capacity positively } \\
\text { associated with a stromal phenotype and negatively correlated with } \\
\text { markers of DNA damage. }\end{array}$ \\
\hline $\begin{array}{l}\text { Targeted } \\
\text { analysis }\end{array}$ & {$[48]$} & 1 & MRI & Multilevel & $\begin{array}{l}\text { Histology and } \\
\text { genomics }\end{array}$ & $\begin{array}{l}\text { Different imaging habitats were related to different growth patterns } \\
\text { when looking at the histopathological examination. Hypoxia and } \\
\text { neovascularisation markers also differ between habitats. } \\
\text { Using presence of somatic mutations and gene copy number variation, } \\
\text { phylogenetic tree reconstruction showed that the habitats derive from } \\
\text { different clones. }\end{array}$ \\
\hline
\end{tabular}

HGSOC with prognostic relevance known as the CLOVAR Classification of Ovarian Cancer were defined-differentiated, immunoreactive, proliferative and mesenchymal [41]. The latter subtype, that is characterised by higher platinum resistance and worse prognosis, establishes the important role of stromal TME in HGSOC [55]. A semantic classification of CT scans based on the peritoneal disease and mesenteric infiltration at diagnosis was found to be associated with the mesenchymal subtype of the CLOVAR classification in 46 patients with HGSOC [44]. The importance of peritoneal involvement in the patients that fall under the mesenchymal subtype was further validated in a multisite cohort of 92 HGSOC patients in which 38 of the patients present in [44] were included [45]. It was also reported that the presence of disease in the pelvis and ovary is associated with the mesenchymal subtype [45].

The proteomic level relevance in HGSOC was shown in the capacity to predict recurrence and survival of the Protein-driven Index of Ovarian Carcinoma (PROVAR), defined using reverse-phase protein arrays in 412 patients from the TCGA [56]. To explore the associations of proteomics and radiomics, a cohort of 20 patients with HGSOC was used together with the expression of proteins involved in amino acid metabolism with highly correlated protein and transcript levels [46]. Imaging traits capturing intra- and inter- site heterogeneity from
CT in a similar manner to [43] were found to be associated with selected proteins such as STXVP2, ASS1 and CBD [46].

Lastly, looking to associations with markers at different levels-genomic, transcriptomic, proteomic and histopathology - a CT-based radiomic signature obtained from 297 HGSOC patients was found to be positively associated with a stromal phenotype, correlated to poor prognosis in different tumour types including ovarian cancer and negatively correlated with markers of DNA damage [47].

Similar radiogenomic associations have also been reported for other cancer types and imaging modalities such as MRI (glioblastoma [57, 58], oligodendrogliomas [59] and breast cancer [60, 61]), CT (lung cancer [62], lung adenocarcinoma [63] and hepatocellular carcinoma [64]) and PET (non-small cell lung cancer [65, 66] and lymphoma [67]). Several reviews have already comprehensively compared these radiogenomic studies and their main limitations $[68,69]$.

These radiogenomic studies suggest important associations between radiomics and tumour biology, but it is important to note that they are generally based on datasets of limited size. Another key limitation is that these studies look for associations between radiomic features that are calculated for the whole lesion (or lesions) whilst the molecular profiling information is obtained from a single biopsy, 
sampling only a small part of the lesion or disease burden. This biopsy is often obtained by an imageguided procedure that provides anatomical information without capturing radiomic phenotypes (Fig. 2, approach 1). This strategy for guiding biopsies is the standard of care in most cancer types, but a single diagnostic biopsy cannot capture the complexity of whole tumours [70]. Likewise, the association of radiomic features with the molecular characteristics studied only in a single region or lesion may be hindering more specific and three-dimensional associations of imaging biomarkers with distinct molecular profiles.

\section{Habitat radiogenomics and targeted biopsies}

Most radiomic analyses rely upon average quantitative features over the whole tumour volume, assuming that the imaging phenotype is the same across the lesion and between metastatic sites and therefore disregarding intratumoural heterogeneity [29]. Nevertheless, images often reveal heterogeneous patterns that can be appreciated quantitatively both in multiparametric functional imaging and in anatomic imaging through texture analysis (Fig. 3) [68]. Regions within a tumour that present a distinct imaging phenotype are called habitats [73, 74]. The presence of different habitats may indicate different

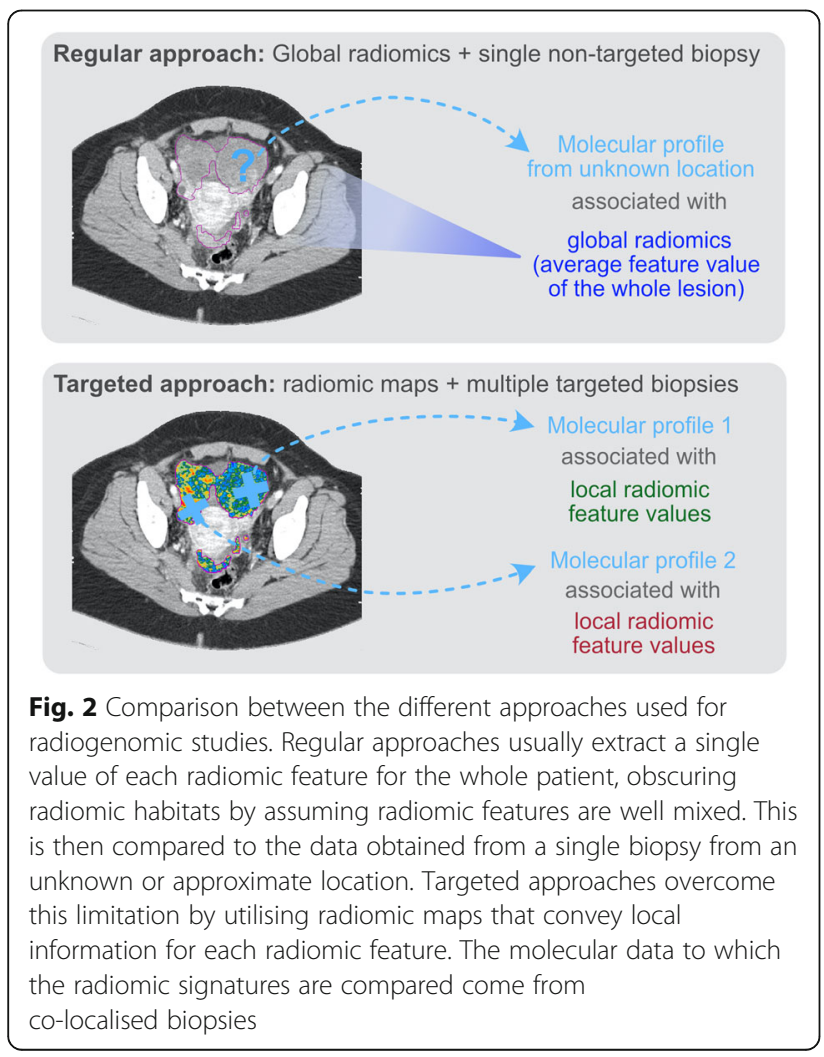

underlying TMEs and tumour molecular profiles. Understanding the biological make-up of tumour habitats could therefore be key to elucidating the mechanisms of tumour evolution and resistance [75].

To date, only a few studies have investigated the relationships between radiomic maps and spatially targeted molecular profiles to describe radiogenomic associations in a local manner. One of the key challenges presented by spatial associations is the accurate co-registration of images with tissue biopsies. Several methods have been proposed, and all of them are based on comparing tissue sections against imaging maps to guide the sampling. To achieve spatial overlap between imaging derived maps and tissue sections, most studies proceed by sectioning a resected specimen parallel to the slices of a pre-surgical scan. This sectioning process can be aided by 3D-printed moulds that ensure a correct alignment of the specimen with the MRI planes. Tissue sectioning can be facilitated further by slots integrated into the moulds that allow for specimen trans-sections parallel to the orientation of imaging slices for improved co-registration. In ovarian cancer, a 3D-printed mould for MRI-guided sampling was tested for a single patient, and areas with different imaging phenotypes obtained by combining perfusion, diffusion and metabolic information were shown to have different histology and genetic compositions [48] (Fig. 4, Table 1).

The mould-guided biopsy approach has recently gained popularity and has been used to investigate the association of radiomic features and histopathology phenotypes in different tumour types, such as prostate cancer [76-79], liver cancer [80] and renal cancer [81]. More recently, updates in the design of these moulds have been proposed to choose the preferred tissue sectioning angle, transforming the images and the corresponding maps [82]. This flexibility makes it easier for patient-specific moulds to be used in the clinical routine without disrupting the usual protocols.

The latest methods for targeting biopsies using radiomic maps have opened up new possibilities for associating radiomics and biological features in a localised manner. This is the first step toward comprehensive data integration and multiscale understanding of tumour complexity and behaviour. Nevertheless, most of the methods presented have two clear limitations: (i) very specialised technologies difficult to integrate in the clinical workflow and (ii) only possible after tumour resection, making it impossible to create multiscale models of tumours that track these associations longitudinally. Some of the most recent 3D mould technologies, such as the one proposed in [82], are designed to address the issue of 


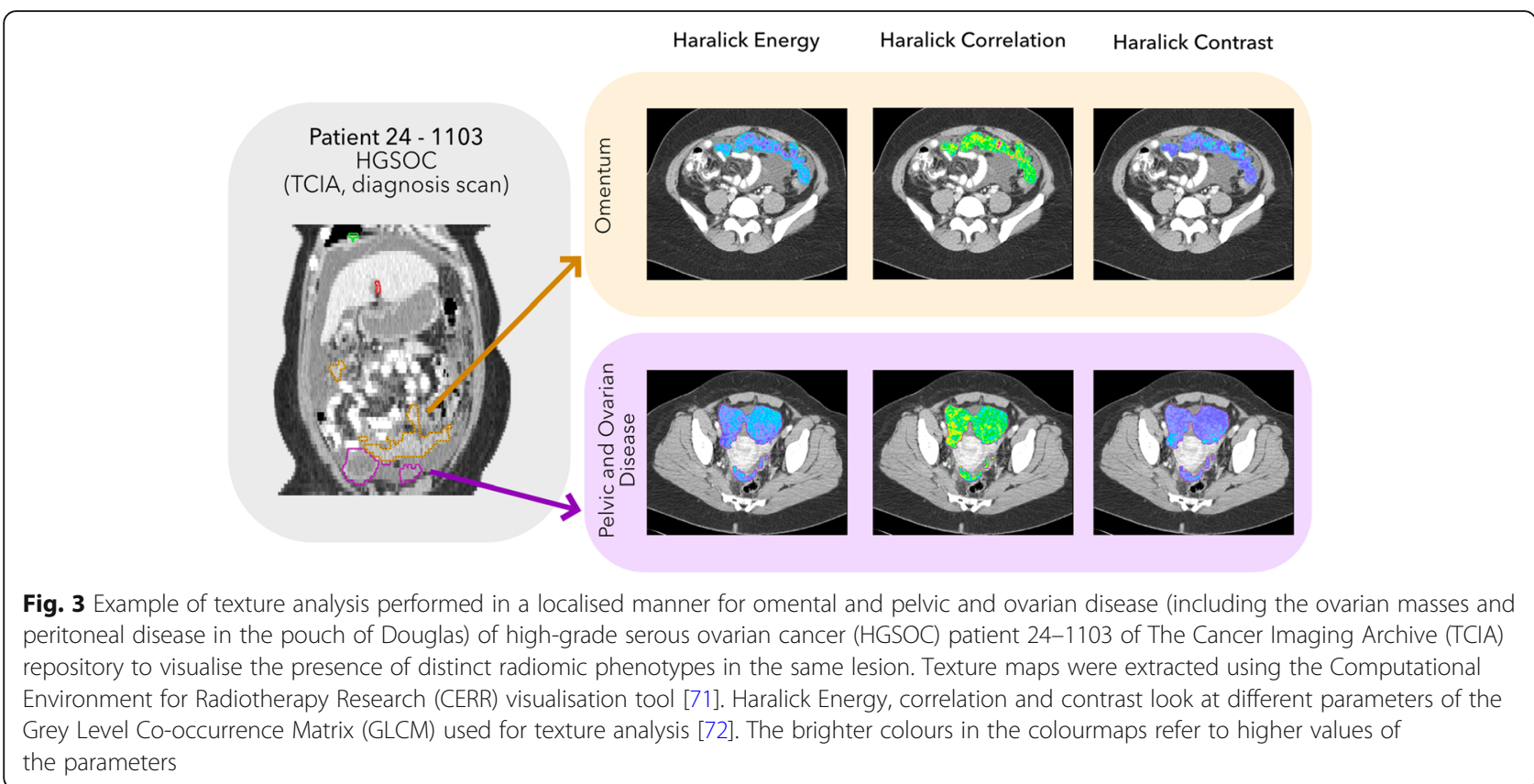

clinical integration and may therefore also provide the key to increasing dataset size.

Accurately, correlating in vivo biopsies with distinct radiomic phenotypes is a different challenge for which fewer solutions exist [22]. A proof of concept based on MRI/US fusion was recently proposed in [83].

\section{The future of radiogenomics: challenges and open questions}

Radiogenomics has emerged as a branch of radiomics that aims to explore associations between imaging phenotypes and biological correlates. Indeed, several studies have been published in different cancer types that support these interrelationships. When spatially colocalised, these studies could facilitate the spatial association of imaging phenotypes and biological pathways, leading to the creation of virtual biopsy maps.

This type of technology is needed in the setting of ovarian cancer to understand tumour evolution and apply this knowledge to clinical management. Detailed knowledge of the molecular tumour profile will enable a more personalised targeted therapy. Current analysis already shows the power of studying the radiogenomics associations in ovarian cancer (Table 1 ). The design and validation of custom $3 \mathrm{D}$ printed moulds to allow radiogenomics information to be integrated is a major key step toward this goal, especially in the surgical scenario. Nevertheless, the design of targeted biopsy methods that can be applied in the clinical setting in patients with HGSOC-using standard of care CT for example-would allow us to track these radiomic changes longitudinally during therapy and ultimately serve as virtual biopsy surrogates to inform clinical decisions.

There are some challenges that need to be carefully addressed before virtual biopsy methods can be integrated into the clinical practice. First, the reproducibility of radiomic features needs to be ensured. The calculation of different features varies between packages, and the numerical values are affected by scan acquisition and reconstruction parameters [33, 84]. Several strategies are in place to address these barriers and to ensure method robustness $[38,85,86]$. Second, the cohort size and high dimensionality of the multiomics datasets to be integrated with the radiology information should be considered in the design of these studies to uncover meaningful associations with significant statistical power. Third, most of the studies on this subject are performed in a retrospective setting. The exploration of these associations in larger and prospective settings is mandatory for clinical translation. Finally, to ensure that these techniques can be utilised routinely in the clinic, targeted sampling methods need to integrate smoothly with clinical practice. For surgical biopsies, this will require robust and automated computational frameworks for 3D-printed mould production. For in vivo biopsies, one of the key developments will be a framework that allows radiologists to visualise radiomic maps in real time as they obtain the biopsy. 


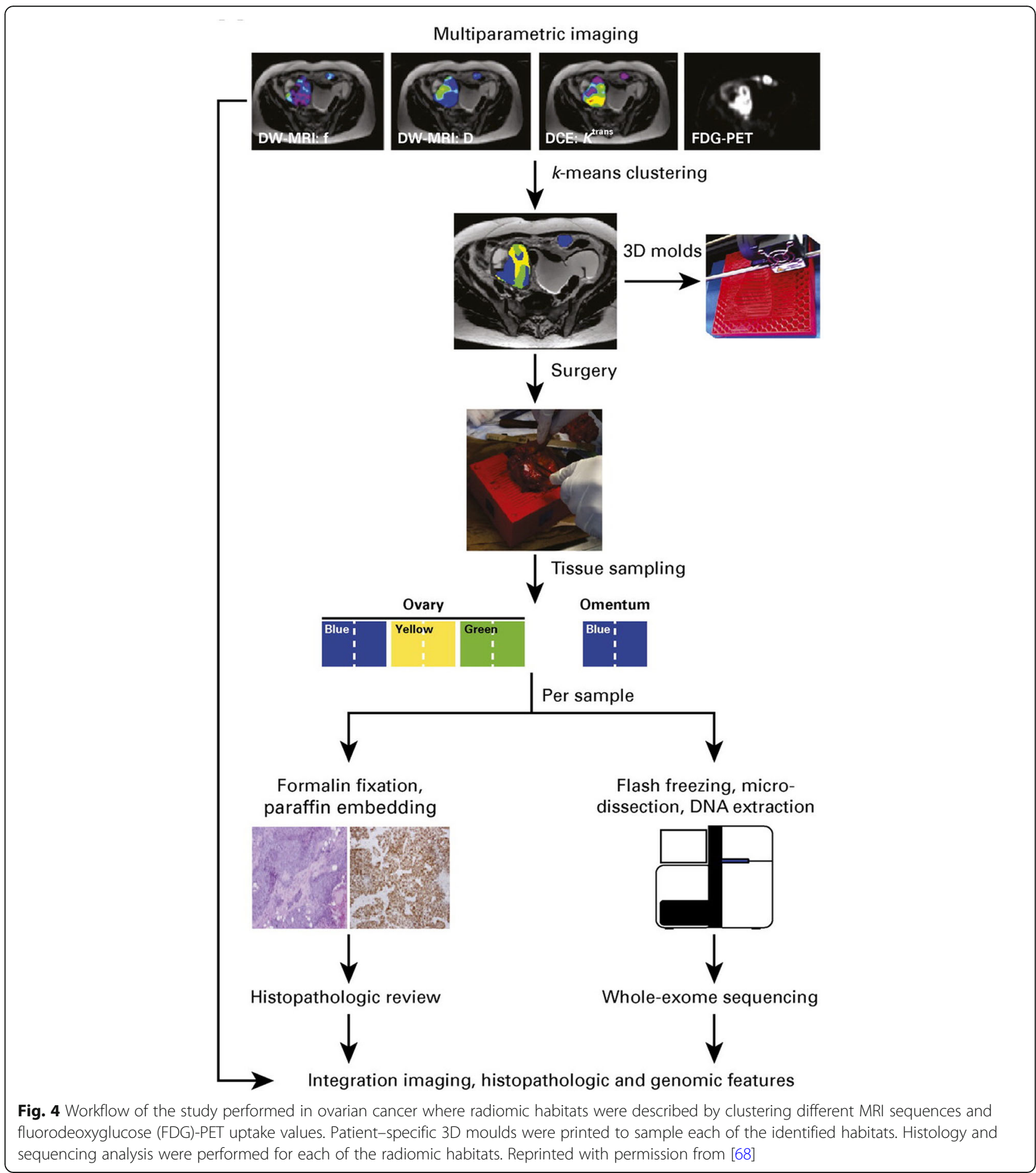

\section{Conclusion}

Targeted radiogenomics could become a powerful addition to the integrative analysis of tumour biology and, thus, a valuable tool in the clinical management of HGSOC patients by predicting molecular profiles from standard of care CT scans. These virtual biopsies could revolutionise the current clinical practice in ovarian cancer, enable the development of biomarkers for treatment selection and detect treatment resistance early during the course of therapy. 


\section{Abbreviations}

CT: Computed tomography; EGFR: Epithelial growth factor receptor: HGSOC: High-grade serous ovarian carcinoma; MRI: Magnetic resonance imaging; NACT: Neoadjuvant chemotherapy; PET: Positron emission tomography; SUV: Standardised uptake value; TME: Tumour microenvironment

\section{Authors' contributions}

PMG performed the literature review. PMG, MCO, FM and ES conceived and drafted the manuscript. LR, MDO, MR, LB, RW, SU, HA and JB revised the manuscript. All authors approved the submitted version.

\section{Funding}

This project has received funding from the European Union's Horizon 2020 research and innovation programme under the Marie Skłodowska-Curie grant agreement No. 766030, the Cancer Research UK Cambridge Institute with core grant C14303/A17197, the Mark Foundation for Cancer Research and Cancer Research UK Cambridge Centre [C9685/A25177]. The sources of funding had no role in the writing of this manuscript, and the views expressed are those of the authors.

\section{Competing interests}

The authors do not have any competing interests.

\section{Author details}

${ }^{1}$ Cancer Research UK Cambridge Institute, University of Cambridge, Cambridge CB2 ORE, UK. ${ }^{2}$ Cancer Research UK Cambridge Centre, University of Cambridge, Cambridge CB2 ORE, UK. ${ }^{3}$ Department of Radiology, University of Cambridge, Cambridge CB2 OQQ, UK. ${ }^{4}$ Department of Biomedical Imaging and Image-guided Therapy, Medical University Vienna, 1090 Vienna, Austria. ${ }^{5}$ Cambridge University Hospitals NHS Foundation Trust, Addenbrooke's Hospital, Cambridge CB2 OQQ, UK

Received: 27 April 2020 Accepted: 16 July 2020

Published online: 17 August 2020

\section{References}

1. Ovarian Cancer, Cancer UK. http://www.cancerindex.org/clink45c.htm.

2. Lisio MA, Fu L, Goyeneche A, Gao ZH, Telleria C (2019) High-grade serous ovarian cancer: basic sciences, clinical and therapeutic standpoints. Int J Mol Sci 20:952

3. Kohn EC, Ivy SP (2017) Whence high-grade serous ovarian cancer. Am Soc Clin Oncol Educ Book 37:443-448 https://doi.org/10.1200/edbk_174718

4. Pokhriyal R, Hariprasad R, Kumar L, Hariprasad G (2019) Chemotherapy resistance in advanced ovarian cancer patients. Biomark Cancer 11: 1179299X1986081 https://doi.org/10.1177/1179299x19860815

5. Bowtell DD, Böhm S, Ahmed AA et al (2015) Rethinking ovarian cancer II: Reducing mortality from high-grade serous ovarian cancer. Nat Rev Cancer 15:668-679

6. Ciriello G, Miller ML, Aksoy BA, Senbabaoglu Y, Schultz N, Sander C (2013) Emerging landscape of oncogenic signatures across human cancers. Nat Genet 45:1127-1133 https://doi.org/10.1038/ng.2762

7. Mishra S, Whetstine JR (2016) Different facets of copy number changes: permanent, transient, and adaptive. Mol Cell Biol 36:1050-1063 https://doi. org/10.1128/mcb.00652-15

8. Stack MS, Nephew KP, Burdette JE, K Mitra A (2018) The Tumor Microenvironment of High Grade Serous Ovarian Cancer. Cancers (Basel) 11(1):21 https://doi.org/10.3390/cancers11010021

9. Labidi-Galy SI, Papp E, Hallberg D et al (2017) High grade serous ovarian carcinomas originate in the fallopian tube. Nat Commun 8:1-11 https://doi. org/10.1038/s41467-017-00962-1

10. Cancer Genome Atlas Research Network (2011) Integrated genomic analyses of ovarian carcinoma. Nature 474:609-615 https://doi.org/10.1038/ nature10166

11. Hoppe MM, Sundar R, Tan DSP, Jeyasekharan AD (2018) Biomarkers for homologous recombination deficiency in cancer. J Natl Cancer Inst 110: 704-713 https://doi.org/10.1093/jnci/djy085

12. Colombo PE, Fabbro M, Theillet C, Bibeau F, Rouanet P, Ray-Coquard I (2014) Sensitivity and resistance to treatment in the primary management of epithelial ovarian cancer. Crit Rev Oncol Hematol 89:207-216
13. Alkema NG, Wisman GB, van der Zee AG, van Vugt MA, de Jong S (2016) Studying platinum sensitivity and resistance in high-grade serous ovarian cancer: different models for different questions. Drug Resist Updat 24:55-69 https://doi.org/10.1016/j.drup.2015.11.005

14. Cunnea P, Stronach EA (2014) Modeling platinum sensitive and resistant high-grade serous ovarian cancer: Development and applications of experimental systems. Front Oncol 4:81

15. Kim S, Han Y, Kim SI, Kim HS, Kim SJ, Song YS (2018) Tumor evolution and chemoresistance in ovarian cancer. NPJ Precis Oncol 2:20 https://doi.org/10. 1038/s41698-018-0063-0

16. Salomon-Perzyński A, Salomon-Perzyńska M, Michalski B, Skrzypulec-Plinta V (2017) High-grade serous ovarian cancer: the clone wars. Arch Gynecol Obstet 295:569-576

17. Kitamura T, Qian BZ, Pollard JW (2015) Immune cell promotion of metastasis. Nat Rev Immunol 15:73-86

18. Vaughan S, Coward Jl, Bast RC Jr et al (2011) Rethinking ovarian cancer: recommendations for improving outcomes. Nat Rev Cancer 11:719

19. Anderson ARA, Weaver AM, Cummings PT, Quaranta V (2006) Tumor morphology and phenotypic evolution driven by selective pressure from the microenvironment. Cell 127:905-915

20. Maley CC, Aktipis A, Graham TA et al (2017) Classifying the evolutionary and ecological features of neoplasms. Nat Rev Cancer 17(10):605-661 https://doi. org/10.1038/nrc.2017.69

21. Gerlinger M, Rowan AJ, Horswell S et al (2012) Intratumor heterogeneity and branched evolution revealed by multiregion sequencing. $\mathrm{N}$ Engl J Med 366:883-892 https://doi.org/10.1056/NEJMoa1113205

22. Sanduleanu S, Woodruff HC, de Jong EEC et al (2018) Tracking tumor biology with radiomics: a systematic review utilizing a radiomics quality score. Radiother Oncol 127:349-360

23. Fotopoulou C, Hall M, Cruickshank D et al (2017) British Gynaecological Cancer Society (BGCS) Epithelial Ovarian / Fallopian Tube / Primary Peritoneal Cancer Guidelines: recommendations for practice. Eur J Obstet Gynecol Reprod Biol 213:123-139

24. SIGN 135 Management of epithelial ovarian cancer. https://www.sign.ac.uk/ sign-135-management-of-epithelial-ovarian-cancer.

25. Ovarian cancer: recognition and initial management guidance, NICE. https:// www.nice.org.uk/guidance/cg122. Accessed 28 Jun 2020

26. Larue RT, Defraene G, De Ruysscher D, Lambin P, Van Elmpt W (2017) Quantitative radiomics studies for tissue characterization: a review of technology and methodological procedures. Br J Radiol 90:20160665 https://doi.org/10.1259/bjr.20160665

27. Aerts HJWL, Velazquez ER, Leijenaar RTH et al (2014) Decoding tumour phenotype by noninvasive imaging using a quantitative radiomics approach. Nat Commun 5 https://doi.org/10.1038/ncomms5006

28. Lambin P, Rios-Velazquez E, Leijenaar $\mathrm{R}$ et al (2012) Radiomics: extracting more information from medical images using advanced feature analysis. Eur J Cancer 48:441-446 https://doi.org/10.1016/j.ejca. 2011.11.036

29. Gillies RJ, Kinahan PE, Hricak H (2016) Radiomics: images are more than pictures, they are data. Radiology 278:563-577 https://doi.org/10.1148/ radiol.2015151169

30. Lambin P, Leijenaar RTH, Deist TM et al (2017) Radiomics: The bridge between medical imaging and personalised medicine. Nat Rev Clin Oncol 14:749-762

31. Papanikolaou N, Matos C, Koh DM (2020) How to develop a meaningful radiomic signature for clinical use in oncologic patients. Cancer Imaging 20:33

32. Rizzo S, Botta F, Raimondi S et al (2018) Radiomics of high-grade serous ovarian cancer: association between quantitative $\mathrm{C} T$ features, residual tumour and disease progression within 12 months. Eur Radiol 28:4849-4859 https://doi.org/10.1007/s00330-018-5389-z

33. Parekh V, Jacobs MA (2016) Radiomics: a new application from established techniques. Expert Rev Precis Med Drug Dev 1:207-226

34. Parmar C, Grossmann P, Bussink J, Lambin P, Aerts HJ (2015) Machine learning methods for quantitative radiomic biomarkers. Sci Rep 5 https:// doi.org/10.1038/srep13087

35. Hosny A, Aerts HJ, Mak RH (2019) Handcrafted versus deep learning radiomics for prediction of cancer therapy response. Lancet Digital Health 1(3):e106-e107

36. Montagnon E, Cerny M, Cadrin-Chênevert A et al (2020) Deep learning workflow in radiology: a primer. Insights Imaging 11:22 
37. Vial A, Stirling D, Field M et al (2018) The role of deep learning and radiomic feature extraction in cancer-specific predictive modelling: a review. Transl Cancer Res 7:803-816 https://doi.org/10.21037/21823

38. Traverso A, Wee L, Dekker A, Gillies R (2018) Repeatability and reproducibility of radiomic features: a systematic review. Int J Radiat Onco Biol Phys 102:1143-1158 https://doi.org/10.1016/j.jirobp.2018.05.053

39. Avanzo M, Stancanello J, El Naqa I (2017) Beyond imaging: the promise of radiomics. Phys Medica 38:122-139

40. Mazurowski MA (2015) Radiogenomics: what it is and why it is important. J Am Coll Radiol 12:862-866 https://doi.org/10.1016/j.jacr.2015.04.019

41. Tothill RW, Tinker AV, George J et al (2008) Novel molecular subtypes of serous and endometrioid ovarian cancer linked to clinical outcome. Clin Cancer Res 14:5198-5208 https://doi.org/10.1158/1078-0432.CCR-08-0196

42. Nougaret S, Lakhman Y, Gönen M et al (2017) High-grade serous ovarian cancer: associations between BRCA mutation status, CT imaging phenotypes, and clinical outcomes. Radiology 285:472-481 https://doi.org/ 10.1148/radiol.2017161697

43. Vargas HA, Veeraraghavan $H$, Micco M et al (2017) A novel representation of inter-site tumour heterogeneity from pre-treatment computed tomography textures classifies ovarian cancers by clinical outcome. Eur Radiol 27:39914001 https://doi.org/10.1007/s00330-017-4779-y

44. Vargas HA, Miccò M, Hong SI et al (2015) Association between morphologic $\mathrm{CT}$ imaging traits and prognostically relevant gene signatures in women with high-grade serous ovarian cancer: a hypothesis-generating study. Radiology 274:742-751 https://doi.org/10.1148/radiol.14141477

45. Vargas HA, Huang EP, Lakhman Y et al (2017) Radiogenomics of high-grade serous ovarian cancer: multireader multi-institutional study from the Cancer Genome Atlas Ovarian Cancer Imaging Research Group. Radiology 285:482492 https://doi.org/10.1148/radiol.2017161870

46. Beer L, Sahin H, Bateman N et al (2020) Integration of proteomics with CTbased qualitative and texture features in high-grade serous ovarian cancer patients: an exploratory analysis. Eur Radiol https:/doi.org/10.17863/CAM.49644

47. Lu H, Arshad M, Thornton A et al (2019) A mathematical-descriptor of tumor-mesoscopic-structure from computed-tomography images annotates prognostic- and molecular-phenotypes of epithelial ovarian cancer. Nat Commun 10:1-11 https://doi.org/10.1038/s41467-019-08718-9

48. Weigelt B, Vargas HA, Selenica P et al (2019) Radiogenomics analysis of intratumor heterogeneity in a patient with high-grade serous ovarian cancer. JCO Precis Oncol:1-9 https://doi.org/10.1200/po.18.00410

49. Liu J, Cristea MC, Frankel P et al (2012) Clinical characteristics and outcomes of BRCA-associated ovarian cancer: genotype and survival. Cancer Genet 205:34-41 https://doi.org/10.1016/j.cancergen.2012.01.008

50. Yang D, Khan S, Sun $Y$ et al (2011) Association of BRCA1 and BRCA2 mutations with survival, chemotherapy sensitivity, and gene mutator phenotype in patients with ovarian cancer. JAMA 306:1557-1565 https://doi. org/10.1001/jama.2011.1456

51. Hyman DM, Zhou Q, lasonos A et al (2012) Improved survival for BRCA2 -associated serous ovarian cancer compared with both BRCA -negative and BRCA1 -associated serous ovarian cancer. Cancer 118:3703-3709 https://doi. org/10.1002/cncr.26655

52. Etemadmoghadam D, Defazio A, Beroukhim R et al (2009) Integrated genome-wide DMA copy number and expression analysis identifies distinct mechanisms of primary chemoresistance in ovarian carcinomas. Clin Cancer Res 15:1417-1427 https://doi.org/10.1158/1078-0432.CCR-08-1564

53. Hwang HC, Clurman BE (2005) Cyclin E in normal and neoplastic cell cycles. Oncogene 24:2776-2786

54. Weinstein JN, Collisson EA, Mills GB et al (2013) The cancer genome atlas pan-cancer analysis project. Nat Genet 45:1113-1120

55. Verhaak RGW, Tamayo P, Yang JY et al (2013) Prognostically relevant gene signatures of high-grade serous ovarian carcinoma. J Clin Invest 123:517525 https://doi.org/10.1172/JCl65833

56. Yang JY, Yoshihara K, Tanaka K et al (2013) Predicting time to ovarian carcinoma recurrence using protein markers. J Clin Invest 123:3740-3750 https://doi.org/10.1172/JCl68509

57. Cui Y, Ren S, Tha KK, Wu J, Shirato H, Li R (2017) Volume of high-risk intratumoural subregions at multi-parametric MR imaging predicts overall survival and complements molecular analysis of glioblastoma. Eur Radiol 27: 3583-3592 https://doi.org/10.1007/s00330-017-4751-X

58. Li Y, Liu X, Xu K et al (2018) MRI features can predict EGFR expression in lower grade gliomas: A voxel-based radiomic analysis. Eur Radiol 28:356-362 https://doi.org/10.1007/s00330-017-4964-z
59. Brown R, Zlatescu M, Sijben A et al (2008) The use of magnetic resonance imaging to noninvasively detect genetic signatures in oligodendroglioma. Clin Cancer Res 14:2357-2362 https://doi.org/10.1158/1078-0432.CCR-07-1964

60. Wu J, Sun $X$, Wang J et al (2017) Identifying relations between imaging phenotypes and molecular subtypes of breast cancer: Model discovery and external validation. J Magn Reson Imaging 46:1017-1027 https://doi.org/10. 1002/jmri.2566

61. Ashraf AB, Daye D, Gavenonis S et al (2014) Identification of intrinsic imaging phenotypes for breast cancer tumors: preliminary associations with gene expression Profiles. Radiology 272:374-384 https://doi.org/10.1148/ radiol.14131375

62. Grossmann P, Stringfield O, El-Hachem N et al (2017) Defining the biological basis of radiomic phenotypes in lung cancer. Elife 6 https://doi.org/10.7554/ eLife. 23421

63. Rios Velazquez E, Parmar C, Liu Y et al (2017) Somatic mutations drive distinct imaging phenotypes in lung cancer. Cancer Res 77:3922-3930 https://doi.org/10.1158/0008-5472.CAN-17-0122

64. Segal E, Sirlin CB, Ooi $C$ et al (2007) Decoding global gene expression programs in liver cancer by noninvasive imaging. Nat Biotechnol 25:675680 https://doi.org/10.1038/nbt1306

65. Yamamoto S, Huang D, Du L et al (2016) Radiogenomic analysis demonstrates associations between ${ }^{18}$ F-fluoro-2-deoxyglucose PET, prognosis, and epithelial-mesenchymal transition in non-small cell lung cancer. Radiology 280:261-270 https://doi.org/10.1148/radiol.2016160259

66. Cottereau AS, Lanic H, Mareschal S et al (2016) Molecular profile and FDGPET/CT Total metabolic tumor volume improve risk classification at diagnosis for patients with diffuse large B-Cell lymphoma. Clin Cancer Res 22:3801-3809 https://doi.org/10.1158/1078-0432.CCR-15-2825

67. Lo Gullo R, Daimiel I, Morris EA, Pinker K (2020) Combining molecular and imaging metrics in cancer: radiogenomics. Insights Imaging 11:1-17

68. Sala E, Mema E, Himoto $Y$ et al (2017) Unravelling tumour heterogeneity using next-generation imaging: radiomics, radiogenomics, and habitat imaging. Clin Radiol 72:3-10

69. Pinker K, Shitano F, Sala E et al (2018) Background, current role, and potential applications of radiogenomics. J Magn Reson Imaging 47:604-620

70. Jamal-Hanjani M, Wilson GA, McGranahan N et al (2017) Tracking the evolution of non-small-cell lung cancer. N Engl J Med 376:2109-2121 https://doi.org/10.1056/NEJMoa1616288

71. Apte AP, lyer A, Crispin-Ortuzar M et al (2018) Technical note: extension of CERR for computational radiomics: a comprehensive MATLAB platform for reproducible radiomics research. Med Phys 45:3713-3720 https://doi.org/10. 1002/mp.13046

72. Haralick RM, Dinstein I, Shanmugam K (1973) Textural features for image classification. IEEE Trans Syst Man Cybern SMC-3:610-621 https://doi.org/10. 1109/TSMC.1973.4309314

73. Gatenby RA, Grove O, Gillies RJ (2013) Quantitative imaging in cancer evolution and ecology. Radiology 269:8-15

74. O'Connor JP, Rose CJ, Waterton JC, Carano RA, Parker GJ, Jackson A (2015) Imaging intratumor heterogeneity: Role in therapy response, resistance, and clinical outcome. Clin Cancer Res 21:249-257

75. McPherson A, Roth A, Laks E et al (2016) Divergent modes of clonal spread and intraperitoneal mixing in high-grade serous ovarian cancer. Nat Genet 48:758-767 https://doi.org/10.1038/ng.3573

76. McGarry SD, Hurrell SL, Iczkowski KA et al (2018) Radio-pathomic maps of epithelium and lumen density predict the location of high-grade prostate cancer. Int J Radiat Oncol Biol Phys 101:1179-1187 https://doi.org/10.1016/j. jirobp.2018.04.044

77. Hurrell SL, McGarry SD, Kaczmarowski A et al (2017) Optimized b-value selection for the discrimination of prostate cancer grades, including the cribriform pattern, using diffusion weighted imaging. J Med Imaging (Bellingham) 5:1 https://doi.org/10.1117/1.jmi.5.1.011004

78. Turkbey B, Mani H, Shah V et al (2011) Multiparametric 3 T prostate magnetic resonance imaging to detect cancer: Histopathological correlation using prostatectomy specimens processed in customized magnetic resonance imaging based molds. J Urol 186:1818-1824 https://doi.org/10. 1016/j.juro.2011.07.013

79. McGarry SD, Bukowy JD, Iczkowski KA et al (2019) Gleason probability maps: a radiomics tool for mapping prostate cancer likelihood in MRI space. Tomography 5:127-134 https://doi.org/10.18383/j.tom.2018.00033

80. Trout AT, Batie MR, Gupta A, Sheridan RM, Tiao GM, Towbin AJ (2017) 3D printed pathological sectioning boxes to facilitate radiological-pathological 
correlation in hepatectomy cases. J Clin Pathol 70:984-987 https://doi.org/ 10.1136/jclinpath-2016-204293

81. Dwivedi DK, Chatzinoff Y, Zhang $Y$ et al (2018) Development of a patientspecific tumor mold using magnetic resonance imaging and 3-dimensional printing technology for targeted tissue procurement and radiomics analysis of renal masses. Urology 112:209-214 https://doi.org/10.1016/j.urology.2017. 08.056

82. Crispin-Ortuzar M, Gehrung M, Ursprung S et al (2019) 3D-printed moulds of renal tumours for image-guided tissue sampling in the clinical setting. bioRxiv:658831 https://doi.org/10.1101/658831

83. Stoyanova R, Pollack A, Takhar M et al (2016) Association of multiparametric MRI quantitative imaging features with prostate cancer gene expression in MRI-targeted prostate biopsies. Oncotarget 7:53362-53376 https://doi.org/ 10.18632/oncotarget.10523

84. Rizzo S, Botta F, Raimondi S et al (2018) Radiomics: the facts and the challenges of image analysis. Eur Radiol Exp 2:36

85. Buckler AJ, Bresolin L, Dunnick NR, Sullivan DC (2011) A collaborative enterprise for multi-stakeholder participation in the advancement of quantitative imaging. Radiology 258:906-914 https://doi.org/10.1148/radiol. 10100799

86. Meyer M, Ronald J, Vernuccio F et al (2019) Reproducibility of CT radiomic features within the same patient: influence of radiation dose and $\mathrm{CT}$ reconstruction settings. Radiology 293:583-591 https://doi.org/10.1148/ radiol.2019190928

\section{Publisher's Note}

Springer Nature remains neutral with regard to jurisdictional claims in published maps and institutional affiliations.

\section{Submit your manuscript to a SpringerOpen ${ }^{\circ}$ journal and benefit from:}

- Convenient online submission

- Rigorous peer review

- Open access: articles freely available online

- High visibility within the field

- Retaining the copyright to your article

Submit your next manuscript at $\boldsymbol{\nabla}$ springeropen.com 